\title{
Formas Orgânicas e Inorgânicas de Mobilização do Cálcio no Solo
}

\author{
Cláudio M. Ziglio, Mario Miyazawa e Marcos A. Pavan* \\ Instituto Agronômico do Paraná (IAPAR), Caixa Postal 481, CEP 86001-970, Londrina, PR.
}

\begin{abstract}
Lack of $\mathrm{Ca}$ and often the presence of Al in subsoil restrict crop-root proliferation. The efficacy of soil surface liming in neutralize subsoil acidity is limited. Laboratory experiments were conducted with leaching columns to evaluate some organic and inorganic techniques to accelerate the mobility of $\mathrm{Ca}$ in a variable charge soil. Two different experiments were conducted, one with $\mathrm{Ca}$-salts: $\mathrm{CaCO}_{3}, \mathrm{CaSO}_{4}, \mathrm{Ca}\left(\mathrm{NO}_{3}\right)_{2}$, Ca-acetate, and Ca-citrate; and another one with $\mathrm{CaCO}_{3}$ plus plant residues: black oat (Avena strigosa), wheat (Triticum aestivum) and leucaena (Leucaena leucocephala). The observations included MKCl exchangeable Ca in 0 to 5, 5 to 10, 10 to 20, 20 to 30, 30 to 40 and 40 to $50 \mathrm{~cm}$ soil depth. The effects of $\mathrm{CaCO}_{3}$ without plant residue was limited to the upper $10 \mathrm{~cm}$ of the profile. The efficacy of Ca-salts on the mobility of $\mathrm{Ca}_{\text {ex }}$ followed the order: $\mathrm{NO}_{3}>$ Acetate $>$ Citrate $>$ $\mathrm{SO}_{4}>\mathrm{CO}_{3}$. Plant residues were highly efficient as Ca-carrier in the following order black oat >leucaena $>$ wheat. Metal-organic complexes were assumed to be responsible for the movement of Ca in the profile associated with lime and plant residues.
\end{abstract}

Key words: Ca-leaching, subsoil acidity, green manure, plant residue.

\section{INTRODUÇÃO}

A acidificação do solo é um processo natural resultado da lixiviação de cátions básicos solúveis (Ca, $\mathrm{Mg}, \mathrm{K})$ seguida pela sua substituição por cátions ácidos ( $\mathrm{H}$ e $\mathrm{Al})$ no complexo de troca catiônica. Nos solos agrícolas, este processo tem sido acelerado principalmente pela adição de certos fertilizantes nitrogenados. A calagem é a prática mais utilizada para o controle da acidez do solo devido ao seu efeito no aumento do $\mathrm{pH}$ e de cátions básicos e na diminuição do Al trocável. Para solos em nosso país, tem sido documentado que o calcário aplicado na superfície do solo apresenta uma mobilidade lenta diminuindo a sua eficiência na redução da acidez subsuperficial (Raij et al., 1982; Pavan et al., 1984). Para a neutralização da acidez nas camadas sub-superficiais do solo são necessárias técnicas especiais, tais como a incorporação mecânica profunda do calcário (Farina \& Channon, 1988), o uso de sais de Ca mais solúveis (Adams et al., 1967; Shainberg et al., 1989) e a utilização de compostos orgânicos (Wright et al., 1985; Watt et al., 1991). O principal objetivo destas técnicas é o de incrementar a concentração de $\mathrm{Ca}$ na zona de crescimento das raízes uma vez que o Ca é imóvel no floema das plantas (Barber, 1984). O movimento de Ca no solo através de compostos orgânicos é de especial interesse devido a alta capacidade de produção de biomassa das plantas utilizadas nos sistemas brasileiros de cultivo (Igue \& Pavan, 1984; Calegari et al., 1993). Entretanto, no Brasil há poucos trabalhos publicados que foram programados para avaliar técnicas de manejo na movimentação de $\mathrm{Ca}$ no perfil do solo. Em geral, os estudos conduzidos no Brasil, concentram-se no uso do gesso para aumentar Ca em profundidade no solo (Ritchey, 1981 e 1987).

O objetivo deste estudo foi avaliar os efeitos de alguns sais orgânicos e inorgânicos de $\mathrm{Ca}$ e de resíduos de plantas na mobilização de $\mathrm{Ca}$ no solo.

\footnotetext{
* Autor para correspondência
} 


\section{MATERIAL E MÉTODOS}

Conduziu-se dois experimentos nos laboratórios do Instituto Agronômico do Paraná (IAPAR) em Londrina utilizando-se solo da camada de 0 a $20 \mathrm{~cm}$ de profundidade de um latossolo vermelho escuro distrófico (LEd). O solo foi seco ao ar, moído e passado em peneira de $2 \mathrm{~mm}$ de diâmetro. As características químicas iniciais do solo foram $\mathrm{pH}\left(\mathrm{CaCl}_{2}\right) 4,3, \mathrm{Ca}, \mathrm{Mg}, \mathrm{Ke}$ Al trocáveis 2,0, 0,40, 0,13 e $0,90 \mathrm{cmol}_{\mathrm{c}} \mathrm{kg}^{-}$ ${ }^{1}$, respectivamente.

\section{Experimento 1. Efeito de sais inorgânicos e orgânicos}

O solo foi transferido para tubos confeccionados com papel filtro de $50 \mathrm{~cm}$ de altura por $3 \mathrm{~cm}$ de diâmetro, preenchidos até a altura de $40 \mathrm{~cm}$, ajustando-se a densidade aparente a aproximadamente $0,9 \mathrm{~g} \mathrm{~cm}^{-3}$. Foram utilizados os seguintes sais: $\mathrm{CaSO}_{4}, \mathrm{CaCO}_{3}, \mathrm{Ca}\left(\mathrm{NO}_{3}\right)_{2}$, Caacetato e Ca-citrato. Os sais de $\mathrm{Ca}$ foram aplicados na superfície do solo na dosagem equivalente a $1,16 \mathrm{Mg} \mathrm{ha}^{-1}$ de $\mathrm{Ca}$ (ou $5 \mathrm{Mg} \mathrm{ha}^{-1}$ de $\mathrm{CaSO}_{4} \cdot 2 \mathrm{H}_{2} \mathrm{O}$ ). Os tubos foram lixiviados com aplicações semanais de $100 \mathrm{ml}$ de água destilada por tubo durante 6 semanas, totalizando $600 \mathrm{ml} /$ tubo (equivalente a $1500 \mathrm{~mm}$ de chuva/ano). No final do período foram coletadas amostras de solo, a cada $5 \mathrm{~cm}$ de profundidade, secas ao ar, moídas e passadas em peneira de $2 \mathrm{~mm}$ de diâmetro. O Ca do solo foi extraído com a solução de $\mathrm{KCl} 1 \mathrm{M}$ na proporção de 1:10 (solo:solução) submetido a 10 minutos de agitação e determinado por espectrofotometria de absorção atômica. Utilizou-se delineamento experimental em blocos ao acaso com três repetições. As análises químicas foram feitas em duplicatas.

\section{Experimento 2. Efeito de resíduos vegetais}

O solo foi transferido para tubos de PVC com 60 $\mathrm{cm}$ de altura por $15 \mathrm{~cm}$ de diâmetro, até a altura de $50 \mathrm{~cm}$ a uma densidade aparente $0,9 \mathrm{~g} \mathrm{~cm}^{-3}$. Foram utilizados os seguintes tratamentos: testemunha $\mathrm{CaCO}_{3}$ sem resíduo vegetal; $\mathrm{CaCO}_{3}$ + resíduo de aveia (Avena strigosa); $\mathrm{CaCO}_{3}+$ resíduo de trigo (Triticum aestivum) e $\mathrm{CaCO}_{3}+$ resíduo de leucena (Leucaena leucocephala). Os materiais de leucena foram coletados quando as plantas apresentavam $150 \mathrm{~cm}$ de altura com o diâmetro dos ramos menor que $10 \mathrm{~mm}$ e os de aveia e trigo no final do ciclo produtivo, após a colheita dos grãos. A tabela 1 apresenta a composição química dos resíduos vegetais. $\mathrm{O}$ calcário e os resíduos vegetais foram aplicados em doses equivalentes a 3 e $40 \mathrm{Mg} \mathrm{ha}{ }^{-1}$, respectivamente. Após a instalação do experimento adicionou-se água destilada semanalmente durante 30 dias, mantendo-se a umidade do solo próxima à capacidade de campo (0.01 MPa). A partir do segundo mês iniciou-se o seguinte programa de irrigação: 1700 e $850 \mathrm{ml}$ de água destilada por tubo (equivalentes à 100 e $50 \mathrm{~mm}$ de chuva, respectivamente) alternadas a cada 15 dias durante 3 meses, totalizando $7650 \mathrm{ml}$ de água destilada/tubo (equivalente a $1500 \mathrm{~mm}$ de chuva/ano).

Tabela 1. Composição química dos resíduos vegetais.

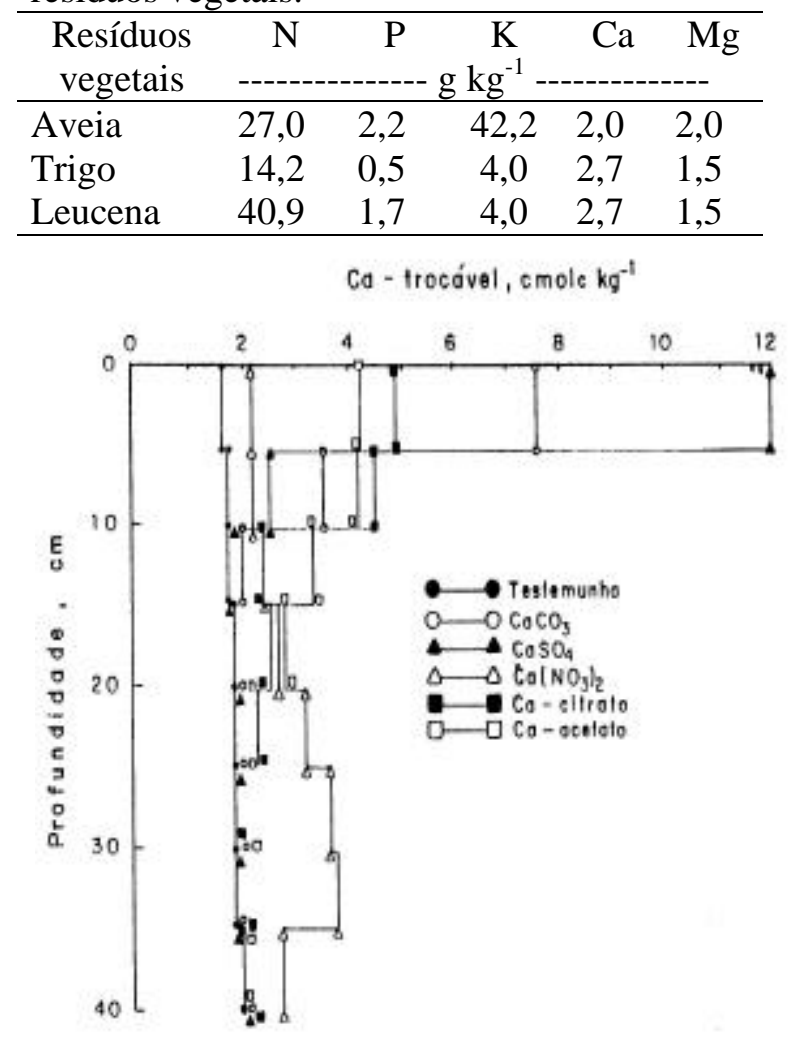

Fiqura 1, Eleito de sais de coíclo no leser de Co-trocónel no perfil do solo.

Após 3 meses, removeram-se os resíduos vegetais remanescentes da superfície do solo e coletaram-se amostras da parte central da 
coluna, nas profundidades de 0 à 5,5 à 10,10 à 20,20 à 30,30 à 40 e 40 à $50 \mathrm{~cm}$. As amostras de solo foram secas ao ar, moídas e passadas em peneira de $2 \mathrm{~mm}$ de diâmetro. Utilizaram-se os mesmos procedimentos descritos em Exp. 1 para extração e determinação de $\mathrm{Ca}$ do solo. Utilizou-se um delineamento experimental em blocos ao acaso com três repetições. As análises químicas foram feitas em duplicatas.

\section{RESULTADOS E DISCUSSÃO}

A figura 1 apresenta os resultados do efeito dos sais de Ca no teor de Ca trocável no solo após os 30 dias de lixiviação. Os sais inorgânicos e orgânicos apresentaram diferentes efeitos na mobilidade do Ca no perfil do solo. A lixiviação do $\mathrm{Ca}$ no solo seguiu a ordem $\mathrm{NO}_{3}>$ Acet. $>$ Citr. $>\mathrm{SO}_{4}>\mathrm{CO}_{3}$. O efeito do $\mathrm{CaCO}_{3}$ no teor de Ca trocável concentrou-se nos primeiros 10 $\mathrm{cm}$ de profundidade. A limitada mobilidade do Ca na forma de carbonato pode ser devida à sua baixa solubilidade (pK 8.32), capacidade de troca de cátions (CTC) dependente do $\mathrm{pH}$ e ausência de um ânion solúvel estável na reação do $\mathrm{CaCO}_{3}$. A reação química do $\mathrm{CaCO}_{3}$ em solo ácido, produz um cátion $\left(\mathrm{Ca}^{++}\right)$e um ânion instável $\left(\mathrm{HCO}_{3}{ }^{-}\right)$que se decompõe em $\mathrm{CO}_{2} \mathrm{e}$ $\mathrm{H}_{2} \mathrm{O}$. O aumento da CTC efetiva pelo $\mathrm{CaCO}_{3}$, diminui a mobilidade do $\mathrm{Ca}$ devido à força de atração do cátion pelas cargas negativas livres no solo. Pavan \& Roth (1992) documentaram em estudo com lisímetro, que a aplicação de calcário na superfície do solo não alterou a concentração de $\mathrm{Ca}^{++}$na solução efluente. Esta característica de baixa mobilidade do $\mathrm{Ca}^{++}$ aplicado na forma de carbonato tem sido documentada em solos brasileiros (Raij et al., 1982; Pavan et al., 1984).

Resultado inesperado foi a baixa mobilidade de $\mathrm{Ca}$ aplicado como $\mathrm{CaSO}_{4}$ (gesso), pois grande número de trabalhos tem demonstrado que este aumenta o teor de Ca no perfil do solo através de vários mecanismos (Pavan et al., 1984; Shainberg et al., 1989; Ritchey, 1981 e 1987). A mobilidade do Ca aplicado como $\mathrm{CaSO}_{4}$ é atribuída à sua maior solubilidade em relação ao carbonato (Bolan et al., 1991). A baixa mobilidade do $\mathrm{Ca}$ como $\mathrm{CaSO}_{4}$ no presente estudo, foi provavelmente devida ao aumento da CTC na camada superficial do solo induzida pelo aumento da força iônica da solução. Takachi \& Pavan (1994) documentaram que a força iônica da solução atua como um tampão da CTC efetiva do solo devido à formação de cargas negativas adicionais na superfície dos colóides. A magnitude deste efeito foi função do tipo de cátion dominante na solução, sendo o $\mathrm{Ca}^{++}$mais efetivo que $\mathrm{Mg}^{++}, \mathrm{K}^{+}$e $\mathrm{Na}^{+}$. Desta forma, o aumento na densidade de cargas negativas induzido pelo $\mathrm{pH}$ e força iônica, aumenta a adsorção do $\mathrm{Ca}^{++}$, diminuindo sua lixiviação para os horizontes inferiores do solo. Esta hipótese é provável devido ao aumento no teor de Ca trocável na superfície do solo $(0-5 \mathrm{~cm})$ com as aplicações de $\mathrm{CaCO}_{3}$ e $\mathrm{CaSO}_{4}$.

Ao contrário do $\mathrm{CaCO}_{3}$, a solubilização do $\mathrm{Ca}\left(\mathrm{NO}_{3}\right)_{2}$ produziu um ânion estável $\mathrm{NO}_{3}^{-}$, o qual promoveu a mobilidade do $\mathrm{Ca}^{++}$como íon acompanhante, para a manutenção da neutralidade química da frente salina no fluxo de massa do solo. Outros estudos também demonstraram a alta eficiência do $\mathrm{Ca}\left(\mathrm{NO}_{3}\right)_{2}$ aplicado na superfície no aumento de $\mathrm{Ca}$ trocável no perfil do solo (Kotze \& Deist, 1975; Pleysier \& Juo, 1981). O uso de $\mathrm{Ca}\left(\mathrm{NO}_{3}\right)_{2}$ como estratégia para incrementar Ca trocável na subsuperfície do solo foi ilustrado por Adams et al. (1967).

Os sais orgânicos de $\mathrm{Ca}$ (acetato e citrato) promoveram uma mobilidade do $\mathrm{Ca}$, superior às obtidas por $\mathrm{CaCO}_{3}$ e $\mathrm{CaSO}_{4}$ e inferior ao $\mathrm{Ca}\left(\mathrm{NO}_{3}\right)_{2}$. Provavelmente, ocorreu a hidrólise do Ca-acetato e Ca-citrato, seguida pela reação de troca com $\mathrm{H}^{+}$e $\mathrm{Al}^{+++}$, causando aumento de Ca-trocável no local das reações e posterior remoção dos cátions ácidos como complexos orgânicos.

A figura 2 apresenta os resultados dos efeitos dos resíduos vegetais na mobilidade do $\mathrm{Ca}$ aplicado como $\mathrm{CaCO}_{3}$ na superfície do solo. A exemplo do exp. 1, o tratamento com aplicação $\mathrm{CaCO}_{3}$ sem resíduos vegetais alterou o teor de Ca trocável apenas na camada de 0 a $5 \mathrm{~cm}$ de profundidade. A adição de resíduos vegetais causou acentuada redução dos teores de Ca- 
trocável nas camadas de 5 a $40 \mathrm{~cm}$ de profundidade. Este resultado sugere que os resíduos vegetais mobilizaram o Ca do calcário e o Ca nativo do complexo de troca do solo para a solução efluente. A ação dos resíduos vegetais na redução dos teores de Ca trocável seguiu a seguinte ordem: aveia $>$ leucena $>$ trigo. A liberação de ânions orgânicos hidrossolúveis durante a mineralização dos resíduos vegetais foi provavelmente a causa da mobilização do $\mathrm{Ca}$ no solo. O menor efeito dos resíduos de trigo pode ter sido devido ao baixo teor de ânions orgânicos, cerca de 5 a $10 \mathrm{Cmol}_{\mathrm{c}}(-) \mathrm{kg}^{-1}$, em sua composição química (Pierre \& Banwart, 1973). Aparentemente, ocorreu a complexação do $\mathrm{Ca}^{++}$ (ácido forte de Lewis) com os grupos funcionais carboxilícos e fenólicos (bases fortes de Lewis)

dos compostos orgânicos. A formação de um complexo neutro $\left(\mathrm{L}^{--} \mathrm{Ca}^{++}\right)^{\circ}$ ou positivamente carregado $\left(\mathrm{LCa}^{-+}\right)^{+}$, onde $\mathrm{L}=$ ligante orgânico, proporcionou a lixiviação do $\mathrm{Ca}^{++}$.

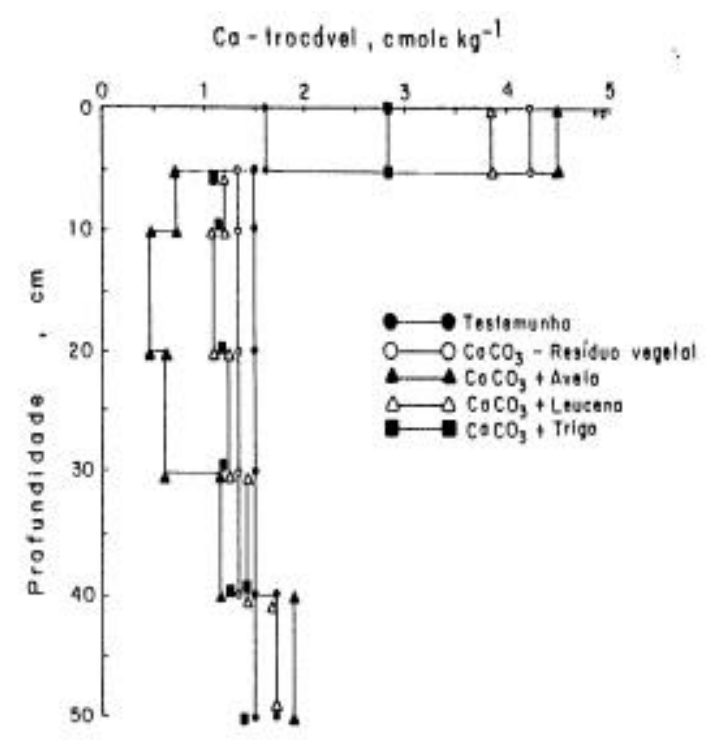

Figura 2. Eleito de residuos vegelals ma mobilitode de Co adicionado $m$ superflitie do sole tomo $\mathrm{CaCO}_{3}$.

Têm sido publicado vários exemplos de tansferência da alcalinidade do calcário para a sub-superfície do solo através da adição de material orgânico na superfície. Wright et al. (1985) demonstraram que o calcário dolomítico (8.1 $\mathrm{Mg} \mathrm{ha}^{-1}$ ) misturado com esterco de bovino (44.8 $\mathrm{Mg} \mathrm{ha}^{-1}$ ) ou EDTA (5.1 Mg ha) promoveu aumentos no $\mathrm{pH}$ e teor de $\mathrm{Ca}$ e diminuiu $\mathrm{Al}$ na sub-superfície do solo, enquanto que o calcário sem adubo orgânico não teve o mesmo efeito. Recentemente, documentou-se que em sistema de intensa reciclagem de resíduos vegetais, como em plantio direto, ocorreu a mobilidade do calcário dolomítico $\left(5,5 \mathrm{Mg} \mathrm{ha}^{-1}\right)$ aplicado na superfície até a profundidade de $60 \mathrm{~cm}$ no solo (Oliveira \& Pavan, 1996). Neste caso, os autores também atribuíram a presença de ligantes orgânicos como responsáveis pelo aumento nos teores de Ca na sub-superfície com aplicação de calcário na superfície do solo em plantio direto. Pavan \& Roth (1992) observaram que a adição de calcário na superfície de um solo coberto com resíduo vegetal (simulando o plantio direto) promoveu maior aumento nas concentrações de íons metálicos na solução lixiviada, comparado com solo descoberto. Os autores atribuíram à reação de complexação dos íons metálicos com compostos orgânicos de baixo peso molecular, a responsabilidade pela maior lixiviação de íons na solução efluente em tal sistema. Blevins et al. (1984) também documentaram maior perda de Ca em sistema de manejo do solo sob plantio direto que sob um sistema de preparo convencional. Novamente, atribuiu-se ao maior teor de matéria orgânica em plantio direto a responsabilidade pela lixiviação do $\mathrm{Ca}$.

\section{CONCLUSÃO}

Os resultados do presente estudo indicaram que a presença de ligantes orgânicos de origem vegetal agem marcadamente sobre a mobilidade do $\mathrm{Ca}$ no solo. O provável efeito negativo da remoção de Ca do solo por compostos orgânicos pode ser minimizado através de rotações de culturas que promovam diferentes capacidades de produção de ânions orgânicos solúveis.

\section{RESUMO}

A falta de $\mathrm{Ca}$ e a presença de $\mathrm{Al}$ na subsuperfície do solo restringem o crescimento das raízes. A eficiência da calagem aplicada na superfície do solo na neutralização da acidez subsuperficial é limitada. Foram conduzidos experimentos de laboratório com colunas de PVC para avaliar técnicas orgânicas e 
inorgânicas na mobilidade do $\mathrm{Ca}$ em solo com carga variável. Foram conduzidos dois experimentos com sais de $\mathrm{Ca}: \mathrm{CaCO}_{3}, \mathrm{CaSO}_{4}$, $\mathrm{Ca}\left(\mathrm{NO}_{3}\right)_{2}$, Ca-acetato e Ca-citrato, e outro com $\mathrm{CaCO}_{3}$ associado com resíduos de aveia preta, trigo e leucena. Avaliaram-se os teores de $\mathrm{Ca}$ trocável em solucão de $\mathrm{KCl} 1 \mathrm{M}$ nas profundidades de 0 a 5,5 a 10,10 a 20,20 a 30 , 30 a 40 e 40 a $50 \mathrm{~cm}$. A eficiência dos sais na mobilidade de $\mathrm{Ca}$ seguiu a ordem: $\mathrm{NO}_{3}>$ Acetado > Citrato $>\mathrm{SO}_{4}>\mathrm{CO}_{3}$. Os resíduos de plantas forma eficientes na mobilização do Ca na seguinte ordem: aveia $>$ leucena $>$ trigo. Atribuiu-se à formação de complexos organometálico a responsabilidade pela mobilização do $\mathrm{Ca}$ no solo associada com a calagem e os resíduos vegetais.

\section{REFERÊNCIAS}

Adams, F.; White, A.W. \& Dawson, R.N (1967). Influence of lime sources and rates on coastal bermudagrass production, soil profile reaction, exchangeable $\mathrm{Ca}$ and $\mathrm{Mg}$. Agron. J., 59:147-149.

Barber, S. (1984). Soil nutrient bioavailability. A mechanist approach. New York, John Wiley \& Sons. 398p.

Blevins, R.L.; Smith, M.S. \& Thomas, G.W. (1984). Changes in soil properties under notillage. In: Phillips, R.E. \& Phillips, S.H. eds. No-tillage agriculture: principles and practices. New York, Van Nostrand Reinhold. p.,1900-230.

Bolan, N.S.; Syers, J.K. \& Sumner, M.E. (1991). Dissolution of various sources of gypsum in aqueous solution and in soil. J. Sci. Food Agric., 57:527-541.

Calegari, A.; Mondardo, M.; Bulisani, E.A.; Wildner, L. Do P.; Costa, M.B.B. da; Alcântara, P.B.; Miyasaka, S.; Amado, T.J.C. (1993). Adubação verde no sul do Brasil. Rio de Janeiro. Assessoria e Serviços à Projetos em Agricultura Alternativa, 346p.

Farina, M.P.W. \& Channon, P. (1988). Acid subsoil amelioration. I. A comparison of several mechanical procedures. Soil Sci. Soc. Am. J., 52:169-175.

Igue, K. \& Pavan, M.A. (1984). Uso eficiente de adubos orgânicos. In: SIMPÓSIO SOBRE FERTILIZANTES NA AGRICULTURA
BRASILEIRA. Brasília 1984. Anais. Brasília, Empresa Brasileira de Pesquisa Agropecuária. p.393-418.

Kotze, W.A.G. \& Deist, J. (1975). Amelioration of subsurface acidity by leaching of surface applied amendments. A laboratory study. Agrochemphysica, 7:39-46.

Oliveira, E.L. de \& Pavan, M.A. (1996). The control of soil acidity in no-till system for soybean production. Soil Till. Res., 38:47-57.

Pavan, M.A. \& Roth, C.H. (1992). Effect of lime and gypsum on chemical composition of runoff and leachate from samples of a Brazilian oxisol. Ci. Cult., 44:391-394.

Pavan, M.A.; Bingham, F.T. \& Pratt, P.F. (1984). Redistribution of exchangeable calcium, magnesium, and aluminum following lime or gypsum applications to a Brazilian Oxisol. Soil Sci. Soc. Am. J., 48:33-38.

Pierre, W.H. \& Banwart, W.L. (1973). Excessbase and excess-base/nitrogen ratio of various crop species and parts of plants. Agron. J., 65:91-96.

Pleysier, J.L. \& Juo, A.S. R. (1981). Leaching of fertilizer ions in a ultisol from the high rainfall tropics. Leaching through undisturbed soil columns. Soil Sci. Soc. Am. J., 45:754-760.

Raij, B. van; Cantarella, H.; Camargo, A.P. De \& Soares, E. (1982). Perdas de cálcio e magnésio durante cinco anos em ensaio de calagem. Rev. bras. Ci. Solo, 6:33-37.

Ritchey, K.D. (1981). Calcium leaching to increase rooting depth in a Brazilian savannah oxisol. Agron. J., 72:40-44.

Ritchey, K.D. (1987). Efficient use of precipitation in humid regions. In: Boersma, L.L.; Elrick, D.E.; Corey, R.B.; Cheng, H.H.; Tucker, T.C. \& Rutteds, E.M. eds. Future developments in soil science research. Madison. Soil Science Society of America. p.327-336.

Shainberg, I.: Sumner, M.E.; Miller, W.P.; Farina, M.P.W. ; Pavan, M.A. \& Fey,. M.Y. (1989). Use of gypsum on soils: a review. Adv. Soil Sci., 9:1-111.

Takachi, C.Y. \& Pavan, M.A. (1994). Cation type and ionic strength induced cation exchange capacity buffering in variable charge soils. Arq. Biol. Tecnol. 37:109-113.

Watt, H. V. H. Van Der; Barnard, R.O.; Cronje, I.J.; Dekker, J.; Croft, G.J.B. \& Walt, M.M. 
van der. (1991). Amelioration of subsoil acidity by application of a coal-derived calcium fulvate to the soil surface. Nature, 350:146-148.

Wright, R.J.; Hern, J.L.; Baligar, V.C. \& Bennett, O.L. (1985). The effect of surface applied soil amendments on barley root growth in acid subsoil. Commun. Soil Sci. Pl. Anal., 16:179-192.
Received: September 24, 1997; Revised: October 15, 1997; Accepted: November 24, 1998. 